Supplementary Information

\title{
The Synthesis and Crystal Structure of As-Synthesized and Calcined Pure Silica Zeolite ITQ-12
}

Xiaobo Yang, Miguel A. Camblor, Yongjae Lee, Haiming Liu, and David H. Olson

\section{Bond lengths and angles of ITQ-12 as refined in the space group $\mathrm{Cm}$}

Table 1: Bond lengths and angles for calcined ITQ-12 as refined in the space group Cm*.

\begin{tabular}{|c|c|c|c|}
\hline Atom pair & Distance $(\AA)$ & Angle & $\left({ }^{\circ}\right)$ \\
\hline Si1-O7 & $1.600(6)$ & O7-Si1-O10 & $99.2(15)$ \\
\hline Si1-O10 & $1.610(6)$ & O7-Si1-O12 & $107.3(18)$ \\
\hline Si1-O12 & $1.616(6)$ & O7-Si1-O15 & $124.1(15)$ \\
\hline \multirow[t]{3}{*}{ Si1-O15 } & $1.607(6)$ & O10-Si1-O12 & $109.8(29)$ \\
\hline & & O10-Si1-O15 & $111.6(19)$ \\
\hline & & O12-Si1-O15 & $104.5(16)$ \\
\hline Si2-O9 & $1.613(6)$ & O9-Si2-O11 & $107.7(20)$ \\
\hline Si2-O11 & $1.603(6)$ & O9-Si2-O13 & $101.2(13)$ \\
\hline $\mathrm{Si2}-\mathrm{O} 13$ & $1.593(6)$ & O9-Si2-O18 & $117.4(21)$ \\
\hline \multirow[t]{3}{*}{ Si2-O18 } & $1.619(6)$ & O11-Si2-O13 & $112.7(15)$ \\
\hline & & O11-Si2-O18 & $104.0(25)$ \\
\hline & & O13-Si2-O18 & $114.0(16)$ \\
\hline Si3-O7 & $1.607(6)$ & O7-Si3-O8 & $109.0(17)$ \\
\hline Si3-O8 & $1.619(6)$ & O7-Si3-O9 & $114.9(13)$ \\
\hline Si3-O9 & $1.612(6)$ & O7-Si3-O14 & $107.5(12)$ \\
\hline \multirow[t]{3}{*}{ Si3-O14 } & $1.605(6)$ & O8-Si3-O9 & 108.1(18) \\
\hline & & O8-Si3-O14 & $108.0(24)$ \\
\hline & & O9-Si3-O14 & $109.2(20)$ \\
\hline Si4-O10 & $1.608(6)$ & O10-Si4-O12 & $108.5(29)$ \\
\hline $\mathrm{Si4}-\mathrm{O} 12$ & $1.617(6)$ & O10-Si4-O13 & 106.4(19) \\
\hline $\mathrm{Si4}-\mathrm{O} 13$ & $1.599(6)$ & O10-Si4-O17 & $116.1(18)$ \\
\hline \multirow[t]{3}{*}{ Si4-O17 } & $1.594(6)$ & O12-Si4-O13 & 109.1(19) \\
\hline & & O12-Si4-O17 & $116.2(21)$ \\
\hline & & O13-Si4-O17 & $99.7(14)$ \\
\hline $\mathrm{Si5-O8}$ & $1.625(6)$ & O8-Si5-O15 & $101.0(17)$ \\
\hline Si5-O15 & $1.602(6)$ & O8-Si5-O19 & $107.9(20)$ \\
\hline Si5-O19 & $1.617(6)$ & O8-Si5-O20 & $110.9(23)$ \\
\hline \multirow[t]{3}{*}{$\mathrm{Si5-O} 20$} & $1.604(6)$ & O15-Si5-O19 & $119.8(15)$ \\
\hline & & O15-Si5-O20 & $100.6(11)$ \\
\hline & & O19-Si5-O20 & $115.6(21)$ \\
\hline
\end{tabular}




\begin{tabular}{|l|l|l|l|}
\hline Si6-O16 & $1.595(6)$ & O16-Si6-O17 & $112.5(12)$ \\
\hline Si6-O17 & $1.595(6)$ & O16-Si6-O18 & $107.6(22)$ \\
\hline Si6-O18 & $1.614(6)$ & O16-Si6-O19 & $112.5(20)$ \\
\hline Si6-O19 & $1.612(6)$ & O17-Si6-O18 & $110.2(18)$ \\
\hline & & O17-Si6-O19 & $100.1(13)$ \\
\hline & & O18-Si6-O19 & $113.9(19)$ \\
\hline & & Si1-O7-Si3 & $142.7(17)$ \\
\hline & & Si3-O8-Si5 & $150.1(23)$ \\
\hline & & Si2-O9-Si3 & $141.0(19)$ \\
\hline & & Si1-O10-Si4 & $163.0(17)$ \\
\hline & & Si2-O11-Si2 & $154.8(32)$ \\
\hline & Si1-O12-Si4 & $158.5(11)$ \\
\hline & Si2-O13-Si4 & $141.8(20)$ \\
\hline & & Si3-O14-Si3 & $156.2(28)$ \\
\hline & Si1-O15-Si5 & $154.3(23)$ \\
\hline & & Si6-O16-Si6 & $133.1(21)$ \\
\hline & & Si4-O17-Si6 & $148.2(21)$ \\
\hline & & Si2-O18-Si6 & $146.7(22)$ \\
\hline & & Si5-O19-Si6 & $151.1(21)$ \\
\hline & & Si5-O20-Si5 & $131.7(23)$ \\
\hline & & &
\end{tabular}

* Soft constraints applied to Si-O distances at $1.610 \AA$ with sigma $=0.001$, overall weight FACTR $=1$ 
Table 2: Bond lengths and angles of as-synthesized ITQ-12 as refined in the space group $\mathrm{Cm}^{*}$.

\begin{tabular}{|c|c|c|c|}
\hline Atom pair & Distance $(\AA)$ & Angle & $\left({ }^{o}\right)$ \\
\hline Si1-O7 & $1.601(6)$ & O7-Si1-O10 & $117.1(16)$ \\
\hline Si1-O10 & $1.608(5)$ & O7-Si1-O12 & $115.1(17)$ \\
\hline Si1-O12 & $1.607(5)$ & O7-Si1-O15 & $97.8(12)$ \\
\hline \multirow[t]{3}{*}{ Si1-O15 } & $1.602(6)$ & O10-Si1-O12 & $104.1(18)$ \\
\hline & & O10-Si1-O15 & $108.5(23)$ \\
\hline & & O12-Si1-O15 & $114.5(15)$ \\
\hline Si2-O9 & $1.620(6)$ & O9-Si2-O11 & $106.6(21)$ \\
\hline Si2-O11 & $1.602(5)$ & O9-Si2-O13 & $111.5(14)$ \\
\hline $\mathrm{Si2}-\mathrm{O} 13$ & $1.608(6)$ & O9-Si2-O18 & $116.7(20)$ \\
\hline \multirow[t]{3}{*}{ Si2-O18 } & $1.627(6)$ & O11-Si2-O13 & $99.3(11)$ \\
\hline & & O11-Si2-O18 & $116.4(26)$ \\
\hline & & O13-Si2-O18 & $105.0(17)$ \\
\hline Si3-O7 & $1.605(6)$ & O7-Si3-O8 & $106.4(17)$ \\
\hline $\mathrm{Si3-O8}$ & $1.618(6)$ & O7-Si3-O9 & $104.9(13)$ \\
\hline Si3-O9 & $1.616(6)$ & O7-Si3-O14 & $111.7(13)$ \\
\hline \multirow[t]{3}{*}{$\mathrm{Si3-O14}$} & $1.605(5)$ & O8-Si3-O9 & $116.9(20)$ \\
\hline & & O8-Si3-O14 & $110.4(25)$ \\
\hline & & O9-Si3-O14 & $106.5(24)$ \\
\hline Si4-O10 & $1.609(5)$ & O10-Si4-O12 & $117.8(19)$ \\
\hline Si4-O12 & $1.608(5)$ & O10-Si4-O13 & $108.8(22)$ \\
\hline Si4-O13 & $1.607(6)$ & O10-Si4-O17 & $100.6(15)$ \\
\hline \multirow[t]{3}{*}{ Si4-O17 } & $1.601(6)$ & O12-Si4-O13 & $100.2(12)$ \\
\hline & & O12-Si4-O17 & $106.6(17)$ \\
\hline & & O13-Si4-O17 & $124.1(13)$ \\
\hline Si5-O8 & $1.621(5)$ & O8-Si5-O15 & $104.1(18)$ \\
\hline Si5-O15 & $1.606(6)$ & O8-Si5-O19 & $109.9(19)$ \\
\hline Si5-O19 & $1.620(6)$ & O8-Si5-O20 & $108.3(24)$ \\
\hline \multirow[t]{3}{*}{ Si5-O20 } & $1.608(5)$ & O15-Si5-O19 & $102.5(12)$ \\
\hline & & O15-Si5-O20 & $112.3(13)$ \\
\hline & & O19-Si5-O20 & $118.6(23)$ \\
\hline Si6-O16 & $1.610(5)$ & O16-Si6-O17 & $102.1(12)$ \\
\hline Si6-O17 & $1.606(6)$ & O16-Si6-O18 & $114.5(28)$ \\
\hline Si6-O18 & $1.621(6)$ & O16-Si6-O19 & $114.8(24)$ \\
\hline \multirow[t]{3}{*}{ Si6-O19 } & $1.622(6)$ & O17-Si6-O18 & $105.4(16)$ \\
\hline & & O17-Si6-O19 & $108.8(14)$ \\
\hline & & O18-Si6-O19 & $110.3(21)$ \\
\hline
\end{tabular}




\begin{tabular}{|c|c|c|c|}
\hline & & & \\
\hline & & Si1-O7-Si3 & $152.6(23)$ \\
\hline & & Si3-O8-Si5 & $141.9(24)$ \\
\hline & & Si2-O9-Si3 & $145.0(18)$ \\
\hline & & Si1-O10-Si4 & $169.7(19)$ \\
\hline & & Si2-O11-Si2 & $135.0(26)$ \\
\hline & & Si1-O12-Si4 & $156.7(11)$ \\
\hline & & Si2-O13-Si4 & $144.3(20)$ \\
\hline & & Si3-O14-Si3 & $142.9(30)$ \\
\hline & & Si1-O15-Si5 & $150.7(24)$ \\
\hline & & Si6-O16-Si6 & $140.4(29)$ \\
\hline & & Si4-O17-Si6 & $143.3(19)$ \\
\hline & & Si2-O18-Si6 & $139.7(22)$ \\
\hline & & Si5-O19-Si6 & $140.3(17)$ \\
\hline & & Si5-O20-Si5 & $143.4(28)$ \\
\hline F1-Si2 & $2.72(4)$ & & \\
\hline F1-Si3 & $2.54(4)$ & & \\
\hline F1-Si5 & $2.56(4)$ & & \\
\hline F1-Si6 & $2.76(4)$ & & \\
\hline $\mathrm{N} 1-\mathrm{C}^{\#}$ & $1.347(5)$ & N1-C2-N3 & 72 \\
\hline N1-C6 ${ }^{\#}$ & $1.482(7)$ & C2-N1-C6 & 144 \\
\hline
\end{tabular}

Soft constraints applied for Si-O distances at $1.610 \AA$ with sigma $=0.001$, overall weight FACTR $=1$.

${ }^{\#}$ Refined translation lengths of the rigid body: V1 $=1.146(4), \mathrm{V} 2=1.482(7) \AA$. 


\section{Closest Interatomic distances between 1,3,4-TMI and}

the ITW $\left[4^{4} 5^{4} 6^{4} 8^{4}\right]$ Cage

Summary of interatomic distances:

\section{$\underline{\text { Ring Atoms }}$}

$\begin{array}{lll}\mathrm{N} 1 & \mathrm{O} 9 & 3.30 \\ \mathrm{~N} 1 & \mathrm{O} 19 & 3.44 \\ & & \\ \mathrm{C} 2 & \mathrm{O} 15 & 3.16 \\ \mathrm{C} 2 & \mathrm{O} 7 & 3.29 \\ & & \\ \mathrm{~N} 3 & \mathrm{O} 9 & 3.42 \\ & & \\ \mathrm{C} 4 & \mathrm{O} 14 & 3.40 \\ \mathrm{C} 4 & \mathrm{O} 20 & 3.40 \\ \mathrm{C} 5 & \mathrm{O} 13 & 3.52\end{array}$

\section{Methyl Groups}

C6 $\quad 013 \quad 3.15$

C6 $018 \quad 3.32$

$\begin{array}{lll}\text { C6 } & \text { O17 } & 3.37\end{array}$

C6 $\quad 019 \quad 3.43$

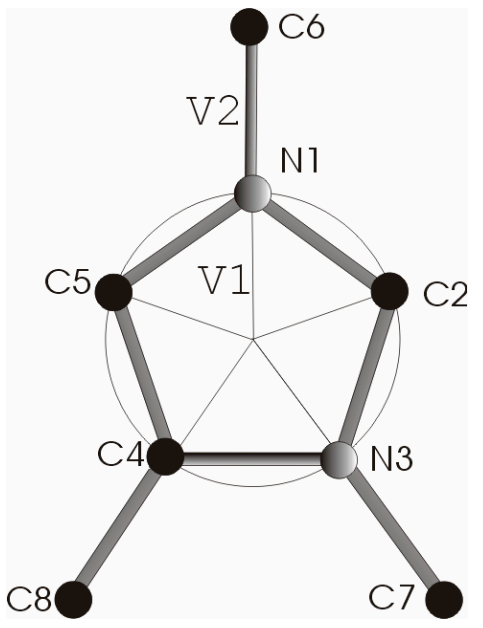

C7 $\quad 015 \quad 3.11$

C7 $\quad$ O9 3.35

$\begin{array}{lll}\mathrm{C} 7 & \mathrm{O} 7 & 3.37\end{array}$

$\begin{array}{lll}\mathrm{C} 8 & \mathrm{O} 13 & 3.42\end{array}$

C8 $\quad 012 \quad 3.45$

Discussion:

The sum of the van der Waals radii for methyl groups and oxygen atoms is $3.40 \AA$, $2.00+1.40^{[1]}$. Methyl groups $\mathrm{C} 6$ and $\mathrm{C} 7$, which are attached to ring nitrogen atoms, have distances significantly shorter than this sum and C8 has distances only $0.02 \AA$ longer, indicating a very good fit of the template molecule. The $\mathrm{C} 7$ methyl group points approximately in the [001] direction while the C6 and C8 methyl groups point approximately in the [010] direction.

The estimated sum of the van der Waals radii for the (aromatic) ring atoms is $3.25 \AA .{ }^{1}$ For the ring atoms the shortest distances are: $\mathrm{N} 1=3.30, \mathrm{C} 2=3.16, \mathrm{~N} 3=3.44 \AA, \mathrm{C} 4=$ 
$3.40, \mathrm{C} 5=3.52$. With the exception of the $\mathrm{C} 2$ atom, all short distances are along [100] and are less than or close to the sum of the Van der Waals radii.

Thus the fit is very close along the [100] and [010] directions and somewhat longer along the channel direction, [001].

[1] L. Pauling, Nature of the Chemical Bond, Cornell University Press, Ithica, NY (1960).

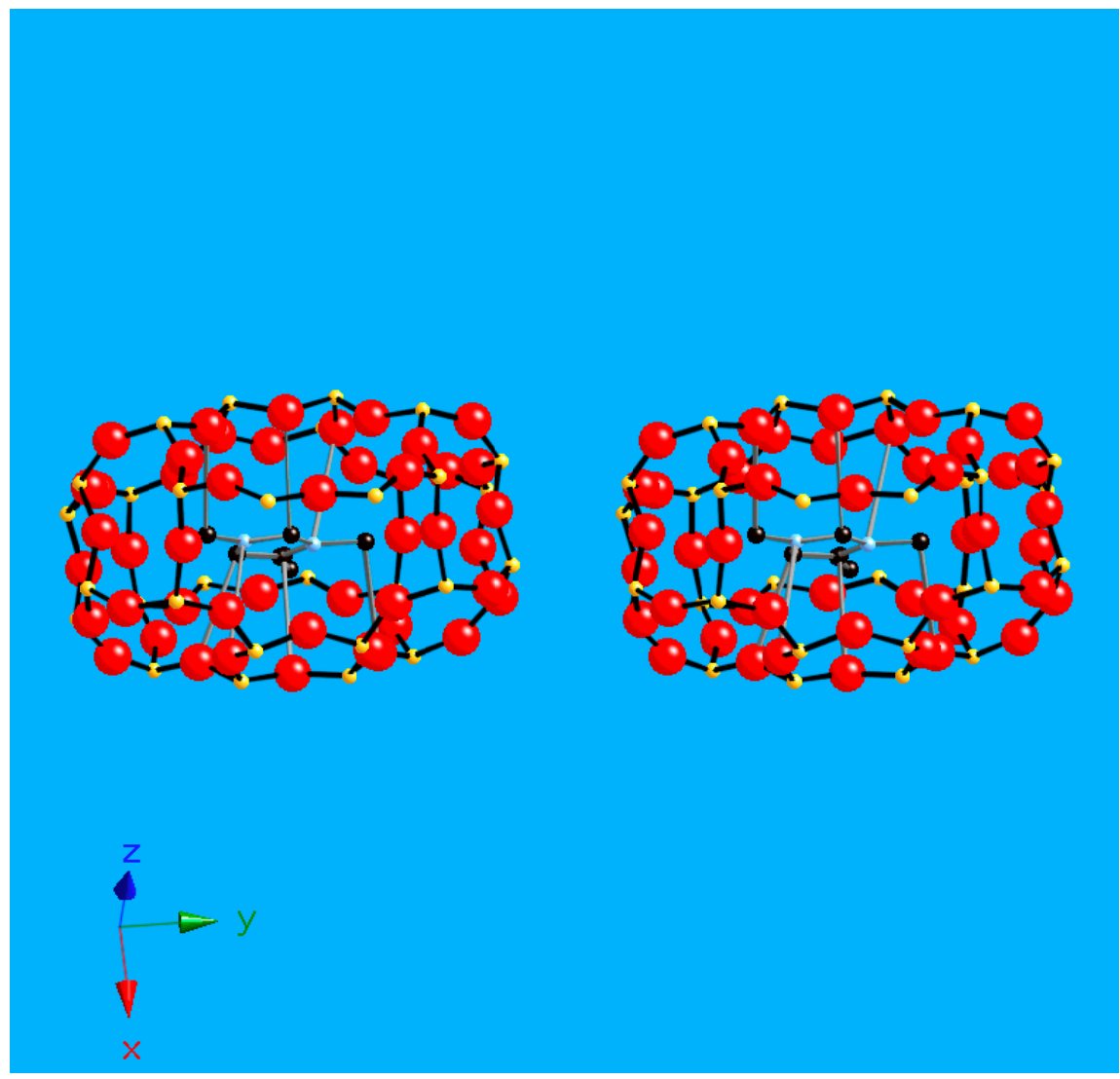




\section{Refinement of calcined ITQ-12 in the space group C2/m}

Table 1: Crystallographic and experimental parameters for Rietveld refinement for calcined ITQ-12 (Model in C2/m symmetry).

\begin{tabular}{|l|l|}
\hline Wavelength & $0.71026 \AA$ \\
\hline Temperature & $298 \mathrm{~K}$ \\
\hline $2 \theta$ range & $0-55^{\circ}\left(\right.$ used $\left.4.25-40^{\circ}\right)$ \\
\hline Step size & $0.01^{\circ}$ \\
\hline No. of data points & 3569 \\
\hline No. of reflections & 707 \\
\hline Space group & $\mathrm{C} 12 / \mathrm{m} 1($ No. 12$)$ \\
\hline Unit cell parameters $(\AA)$ & \\
\hline$a$ & $10.3359(4)$ \\
\hline$b$ & $15.0175(6)$ \\
\hline$c$ & $8.8638(4)$ \\
\hline$\beta$ & $105.356(3)$ \\
\hline Cell Volume $\left(\AA^{3}\right)$ & $1324.7(1)$ \\
\hline Residuals & \\
\hline Rwp & 0.0581 \\
\hline Rp & 0.0445 \\
\hline $\mathrm{R}_{\mathrm{F}}^{2}$ & 0.1047 \\
\hline reduced $\chi^{2}$ & 43.65 \\
\hline & \\
\hline
\end{tabular}


Table 2: Atomic positions and isotropic temperature displacement factors for calcined ITQ-12 in the space group $\mathrm{C} 2 / \mathrm{m}$.

\begin{tabular}{|l|l|l|l|l|l|}
\hline Name & $\mathrm{x}$ & $\mathrm{y}$ & $\mathrm{z}$ & Uiso*100 & Occupancy \\
\hline Si1 & $0.6486(4)$ & $0.24419(35)$ & $0.1082(5)$ & $0.92(10)$ & 1 \\
\hline Si2 & $0.6957(5)$ & $0.39971(35)$ & $0.6684(7)$ & $0.92(10)$ & 1 \\
\hline Si3 & $0.6000(5)$ & $0.39893(33)$ & $0.3085(6)$ & $0.92(10)$ & 1 \\
\hline O4 & 0.5 & $0.2210(9)$ & 0 & $1.41(14)$ & 1 \\
\hline O5 & $0.8072(10)$ & $0.3381(6)$ & $0.7758(12)$ & $1.41(14)$ & 1 \\
\hline O6 & $0.6205(16)$ & 0.500000 & $0.2672(17)$ & $1.41(14)$ & 1 \\
\hline O7 & $0.6569(11)$ & $0.3365(5)$ & $0.1964(12)$ & $1.41(14)$ & 1 \\
\hline O8 & $0.4417(7)$ & $0.3801(7)$ & $0.2808(13)$ & $1.41(14)$ & 1 \\
\hline O9 & $0.6797(10)$ & $0.3767(6)$ & $0.4863(7)$ & $1.41(14)$ & 1 \\
\hline O10 & 0.75 & 0.25 & 0 & $1.41(14)$ & 1 \\
\hline O11 & $0.7394(14)$ & 0.5 & $0.7134(19)$ & $1.41(14)$ & 1 \\
\hline
\end{tabular}


Table 3: Atomic positions and isotropic temperature displacement factors for calcined ITQ-12 in the space group $\mathrm{C} 2 / \mathrm{m}^{*}$.

\begin{tabular}{|c|c|c|c|}
\hline Atom pair & Distance $(\AA)$ & Angle & $\left({ }^{0}\right)$ \\
\hline Si1-O4 & $1.619(5)$ & O4-Si1-O5 & $106.3(6)$ \\
\hline Si1-O5 & $1.595(5)$ & O4-Si1-O7 & $113.5(8)$ \\
\hline Si1-O7 & $1.583(5)$ & O4-Si1-O10 & $109.0(3)$ \\
\hline \multirow[t]{3}{*}{ Si1-O10 } & $1.599(4)$ & O5-Si1-O7 & $113.0(6)$ \\
\hline & & O5-Si1-O10 & $107.9(5)$ \\
\hline & & O7-Si1-O10 & $106.9(4)$ \\
\hline Si2-O5 & $1.584(5)$ & O5-Si2-O8 & $106.1(7)$ \\
\hline Si2-O8 & $1.627(5)$ & O5-Si2-O9 & $109.9(6)$ \\
\hline Si2-O9 & $1.615(5)$ & O5-Si2-O11 & $106.8(6)$ \\
\hline \multirow[t]{3}{*}{$\mathrm{Si2}-\mathrm{O} 11$} & $1.593(5)$ & O8-Si2-O9 & $111.8(6)$ \\
\hline & & O8-Si2-O11 & $108.2(8)$ \\
\hline & & O9-Si2-O11 & $113.6(8)$ \\
\hline Si3-O6 & $1.589(5)$ & O6-Si3-O7 & $109.0(6)$ \\
\hline Si3-O7 & $1.587(6)$ & O6-Si3-O8 & $108.9(9)$ \\
\hline $\mathrm{Si3-O8}$ & $1.614(5)$ & O6-Si3-O9 & $111.1(7)$ \\
\hline \multirow[t]{11}{*}{ Si3-O9 } & $1.608(5)$ & O7-Si3-O8 & $109.3(6)$ \\
\hline & & O7-Si3-O9 & $108.2(6)$ \\
\hline & & O8-Si3-O9 & $110.4(6)$ \\
\hline & & Si1-O4-Si1 & $155.2(11)$ \\
\hline & & Si1-O5-Si2 & $151.4(8)$ \\
\hline & & Si3-O6-Si3 & $145.7(11)$ \\
\hline & & Si1-O7-Si3 & $147.8(8)$ \\
\hline & & Si2-O8-Si3 & $148.3(8)$ \\
\hline & & Si2-O9-Si3 & $145.6(7)$ \\
\hline & & Si1-O10-Si1 & $180.0(0)$ \\
\hline & & Si2-O11-Si2 & $142.1(12)$ \\
\hline
\end{tabular}

* Soft constraints applied for Si-O distances at $1.610 \AA$, with sigma $=0.001$, overall weight $\mathrm{FACTR}=1$. 


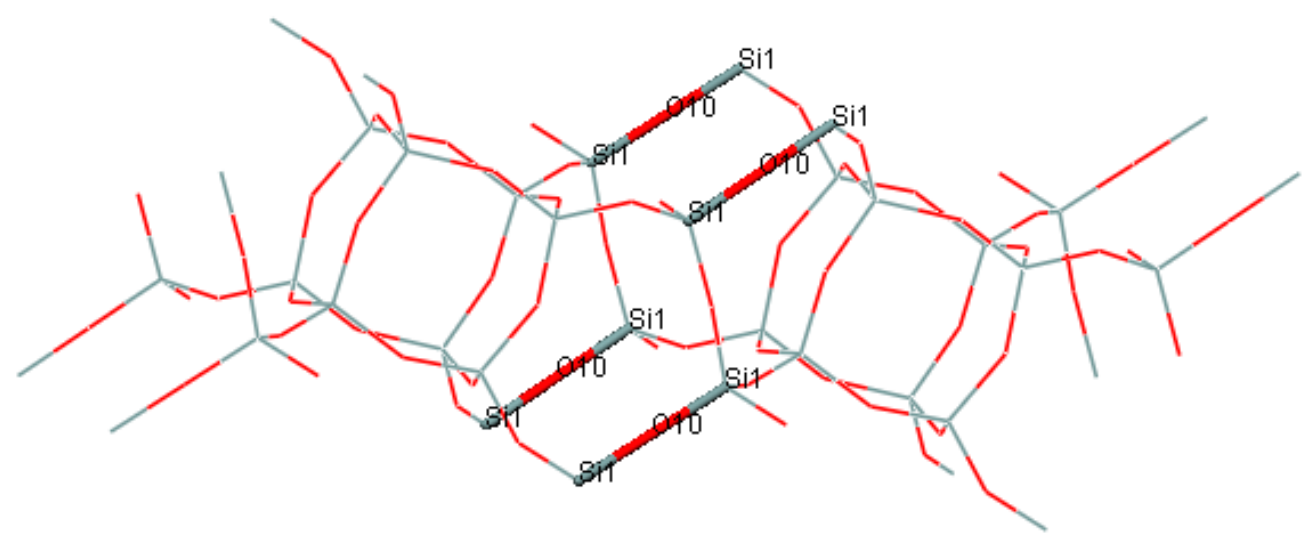

Fig.1: A fragment of calcined ITQ-12 structure as refined in space group C2/m using synchrotron X-ray diffraction data, showing the unrealistic Si-O-Si bonds. 


\section{Refinement of as-synthesized ITQ-12 in the space group C2/m}

Table 1: Crystallographic and experimental parameters for Rietveld refinement for assynthesized ITQ-12 (Model in C2/m symmetry).

\begin{tabular}{|l|l|}
\hline Wavelength & $0.71026 \AA$ \\
\hline Temperature & $298 \mathrm{~K}$ \\
\hline $2 \theta$ range & $0-55^{\circ}\left(\right.$ used $\left.4.25-40^{\circ}\right)$ \\
\hline Step size & $0.01^{\circ}$ \\
\hline No. of data points & 3574 \\
\hline No. of reflections & 691 \\
\hline Space group & $\mathrm{C} 12 / \mathrm{m} 1$ (No. 12) \\
\hline Unit cell parameters $(\AA)$ & \\
\hline$a$ & $10.4480(3)$ \\
\hline$b$ & $14.9855(4)$ \\
\hline$c$ & $8.8367(3)$ \\
\hline$\beta$ & $105.937(2)$ \\
\hline Cell Volume $\left(\AA^{3}\right)$ & $1330.37(7)$ \\
\hline Residuals & \\
\hline Rwp & 0.0527 \\
\hline Rp & 0.0408 \\
\hline $\mathrm{R}_{\mathrm{F}}^{2}$ & 0.0777 \\
\hline reduced $\chi^{2}$ & 31.56 \\
\hline & \\
\hline
\end{tabular}


Table 2: Atomic positions and isotropic temperature displacement factors for assynthesized ITQ-12 in the space group C $2 / \mathrm{m}^{*}$.

\begin{tabular}{|l|l|l|l|l|l|}
\hline Name & $\mathrm{x}$ & $\mathrm{y}$ & $\mathrm{Z}$ & Uiso*100 & Occupancy \\
\hline F1 & 0.5 & 0.5 & 0.5 & $1.02(10)$ & 1 \\
\hline $\mathrm{N} 1$ & $0.4922(35)$ & $0.0842(15)$ & $0.4759(25)$ & $1.39(10)$ & 0.25 \\
\hline C2 & $0.5084(44)$ & $-0.0005(16)$ & $0.5274(22)$ & $0.26(9)$ & 0.25 \\
\hline N3 & $0.4731(30)$ & $-0.0545(15)$ & $0.4016(22)$ & $1.39(10)$ & 0.25 \\
\hline C4 & $0.4349(16)$ & $-0.0033(16)$ & $0.2725(19)$ & $0.26(9)$ & 0.25 \\
\hline C5 & $0.4467(25)$ & $0.0824(15)$ & $0.3184(24)$ & $0.26(9)$ & 0.25 \\
\hline C6 & $0.5196(51)$ & $0.1654(18)$ & $0.5757(34)$ & $0.26(9)$ & 0.25 \\
\hline C7 & $0.4756(46)$ & $-0.1535(16)$ & $0.4049(32)$ & $0.26(9)$ & 0.25 \\
\hline C8 & $0.3879(34)$ & $-0.0357(20)$ & $0.1078(21)$ & $0.26(9)$ & 0.25 \\
\hline Si1 & $0.64841(34)$ & $0.24419(27)$ & $0.1076(4)$ & $0.63(8)$ & 1 \\
\hline Si2 & $0.69289(40)$ & $0.39998(30)$ & $0.6709(5)$ & $0.63(8)$ & 1 \\
\hline Si3 & $0.59463(42)$ & $0.39892(29)$ & $0.3107(5)$ & $0.63(8)$ & 1 \\
\hline O4 & 0.5 & $0.2220(8)$ & 0 & $0.94(12)$ & 1 \\
\hline O5 & $0.8083(8)$ & $0.3367(5)$ & $0.7727(9)$ & $0.94(12)$ & 1 \\
\hline O6 & $0.6173(12)$ & 0.5 & $0.2644(14)$ & $0.94(12)$ & 1 \\
\hline O7 & $0.6526(9)$ & $0.3366(4)$ & $0.1977(9)$ & $0.94(12)$ & 1 \\
\hline O8 & $0.4384(6)$ & $0.3728(5)$ & $0.2735(10)$ & $0.94(12)$ & 1 \\
\hline O9 & $0.6801(8)$ & $0.3743(5)$ & $0.4886(6)$ & $0.94(12)$ & 1 \\
\hline O10 & 0.75 & 0.25 & 0 & $0.94(12)$ & 1 \\
\hline O11 & $0.7409(12)$ & 0.5 & $0.7172(14)$ & $0.94(12)$ & 1 \\
\hline
\end{tabular}

* Soft constraints applied for Si-O distances at $1.610 \AA$, with sigma $=0.001$, overall weight FACTR $=1$. 
Table 3: Atomic positions and isotropic temperature displacement factors for assynthesized ITQ-12 in the space group $\mathrm{C} 2 / \mathrm{m}^{*}$.

\begin{tabular}{|c|c|c|c|}
\hline Atom pair & Distance $(\AA)$ & Angle & $\left(^{\circ}\right)$ \\
\hline Si1-O4 & $1.615(4)$ & O4-Si1-O5 & $106.6(5)$ \\
\hline Si1-O5 & $1.591(5)$ & O4-Si1-O7 & $111.3(6)$ \\
\hline Si1-O7 & $1.593(5)$ & O4-Si1-O10 & $110.20(22)$ \\
\hline \multirow[t]{3}{*}{ Sil-O10 } & $1.610(3)$ & O5-Si1-O7 & $111.5(5)$ \\
\hline & & O5-Si1-O10 & $108.7(4)$ \\
\hline & & O7-Si1-O10 & $108.43(35)$ \\
\hline Si2-O5 & $1.602(5)$ & O5-Si2-O8 & $104.4(5)$ \\
\hline Si2-O8 & $1.630(4)$ & O5-Si2-O9 & $105.1(5)$ \\
\hline Si2-O9 & $1.626(5)$ & O5-Si2-O11 & $106.0(5)$ \\
\hline \multirow[t]{3}{*}{$\mathrm{Si2}-\mathrm{O} 11$} & $1.598(4)$ & O8-Si2-O9 & $113.1(5)$ \\
\hline & & O8-Si2-O11 & $112.9(6)$ \\
\hline & & O9-Si2-O11 & $114.2(6)$ \\
\hline Si3-O6 & $1.603(4)$ & O6-Si3-O7 & $106.5(5)$ \\
\hline $\mathrm{Si3}-\mathrm{O} 7$ & $1.602(5)$ & O6-Si3-O8 & $112.8(7)$ \\
\hline Si3-O8 & $1.622(5)$ & O6-Si3-O9 & $112.5(6)$ \\
\hline \multirow[t]{11}{*}{ Si3-O9 } & $1.623(5)$ & O7-Si3-O8 & $105.4(5)$ \\
\hline & & O7-Si3-O9 & $105.6(5)$ \\
\hline & & O8-Si3-O9 & $113.4(5)$ \\
\hline & & Si1-O4-Si1 & $156.3(9)$ \\
\hline & & Si1-O5-Si2 & $149.0(7)$ \\
\hline & & Si3-O6-Si3 & $141.7(9)$ \\
\hline & & Si1-O7-Si3 & $148.8(6)$ \\
\hline & & Si2-O8-Si3 & $139.6(5)$ \\
\hline & & Si2-O9-Si3 & $141.2(5)$ \\
\hline & & Si1-O10-Si1 & $180.0(0)$ \\
\hline & & $\mathrm{Si} 2-\mathrm{O} 11-\mathrm{Si} 2$ & $139.5(9)$ \\
\hline F1-Si2 & $2.630(4)$ & & \\
\hline F1-Si3 & $2.641(4)$ & & \\
\hline $\mathrm{N} 1-\mathrm{C} 2^{\#}$ & $1.342(5)$ & N1-C2-N3 & 72 \\
\hline $\mathrm{N} 1-\mathrm{C}^{\#}$ & $1.483(6)$ & C2-N1-C6 & 144 \\
\hline
\end{tabular}

* Soft constraints applied for Si-O distances at $1.610 \AA$, with sigma $=0.001$, overall weight $\mathrm{FACTR}=1$.

\# Refined translation lengths of the rigid body: V1=1.141(4), V2 = 1.483(6) $\AA$. 